\title{
Unraveling the Complex Architecture of Hybrid Hard-soft Polymer-Nanoparticle Systems with Soft Microscopy
}

\author{
Chamille Lescott, Mallika Modak, Evan Scott and Vinayak Dravid \\ ${ }^{1}$ Northwestern University, Evanston, Illinois, United States
}

Self-assembled polymer nanostructures hold promise as drug delivery vehicles for the treatment of many diseases. As soft materials, polymer nanostructures present characterization challenges, such as beam sensitivity, low contrast for electron microscopy, and structural instability upon drying. Therefore, the analysis of soft material structure is often limited to cryogenic microscopy, but characterization by standard energy dispersive spectroscopy (EDS) and electron energy loss spectroscopy is difficult to implement due to the vitreous ice melting during data acquisition. The incorporation of "hard" nanoparticles into polymer structures adds an additional challenge due to the differing characterization considerations of the two material systems. Here we investigate a unique self-assembling polymer encapsulating iron-based magnetic nanostructures (MNS) as an example system for soft microscopy analysis. Scanning transmission electron microscopy (STEM) and the use of elemental mapping via EDS allow us to probe the nature of the hard-soft interface between the polymer and the inorganic nanoparticles. The elucidated form of these polymer particles gives insight into the in vivo particle degradation mechanism that drives drug delivery.

The self-assembled polymer structures investigated in this work are magnetic nanostructure- loaded socalled bicontinuous nanospheres (MNS-BCNs). Diblock copolymer, poly(ethylene glycol)17poly(propylene sulfide) 17 (PEG- $b$-PPS), self-assembles into biocontinuous nanospheres (BCNs) when exposed to water via flash-nanoprecipitation[1] [2]. Hydrophobic small molecules and $4 \mathrm{~nm}$ MNS may be incorporated into the structure during the particle formation process. The MNS serve as contrast agents for magnetic resonance imaging, providing additional functionality to MNS-BCNs. Here we aim to connect the structure of the MNS-BCN to the particle formation and degradation mechanism.

To prepare the sample for analytical microscopy techniques such as EDS, a solution of MNS-BCNs in $0.4 \%$ methyl cellulose was deposited on a hydrophilized TEM grid. The addition of methyl cellulose prevents polymer structures from lysing [3]. The concentration of the MNS-BCNs in methyl cellulose was optimized to avoid the formation of particle aggregates. Further, the samples were imaged on a Hitachi2300 dedicated STEM operated at $200 \mathrm{kV}$. STEM allows for direct interpretation through the image contrast and shows that the morphology of the BCNs change with varying levels of MNS loading (Figure 1). The MNS forms an aggregate inside the BCN that differs in size based on the amount of MNS incorporated during flash-nanoprecipitation.

The sulfur in the backbone of the PEG-PPS serves as an elemental marker of the polymer. Elemental maps were formed using the iron $(6.405 \mathrm{keV})$ and sulfur $(2.309 \mathrm{keV})$

K edge. A quantitative line scan of the EDS map reveals that the sulfur signal and, thus the polymer concentration increases around the aggregate of MNS (Figure 2b). The EDS results suggest a mechanism for MNS-BCN assembly. It seems as if polymer surrounds and stabilizes the mass of MNS then continues to form the cubically ordered channels characteristic of BCNs. These BCN structures break apart when exposed to oxidative conditions. Oxidation increases the hydrophilicity of the polypropylene sulfide in the diblock copolymer by converting it into sulfoxide and sulfone derivatives, which disrupts the ratio of 
hydrophobic and hydrophilic interactions that stabilize the assembled structure [4] . The MNS-BCNs then break down, forming MNS aggregates stabilized by polymer (Figure 2c). Through quantitative EDS mapping, we now understand that the MNS in the MNS-BCN are encased in polymer, allowing for the formation of smaller MNS-polymer aggregates as the BCN degrades. These results are consistent with a recent report of a bicontinuous to micellar transition in nanostructure to occur following oxidation of PEG$b$-PPS BCNs [4].

Soft microscopy is an expanding field focused on a combination of relevant analytical techniques, appropriate sample preparations and targeted analysis that helps us understand the form of structures like the MNS-BCN. We aim to show more extensive work documenting the variation of the sulfur signal relative to the iron signal in MNS-BCNs loaded with different amounts of MNS. We will utilize quantitative EDS to determine how the concentration of loaded therapeutics varies across the structure of the BCNs. MNS-BCNs serve as a basis for studying other composites of self-assembled polymer and metal nanoparticles. Additionally, we will show the transferability of these techniques to similar materials systems to highlight the broad implications soft microscopy may have on our understanding of material structure [5].
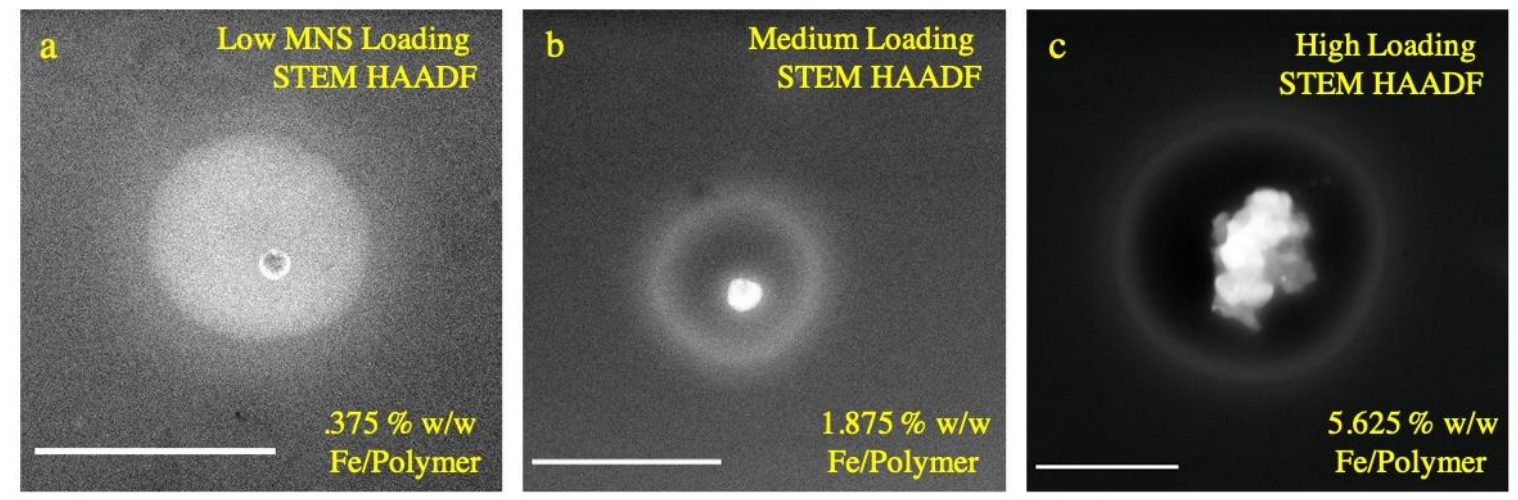

Figure 1. Scale bar: $1 \mathrm{~mm}$. STEM HAADF images of MNS-BCNs embedded in methyl cellulose. The level of MNS loading increases from (a) to (c). The iron concentration was determined by inductively coupled plasma mass spectrometry and represented as a percentage of the polymer mass.
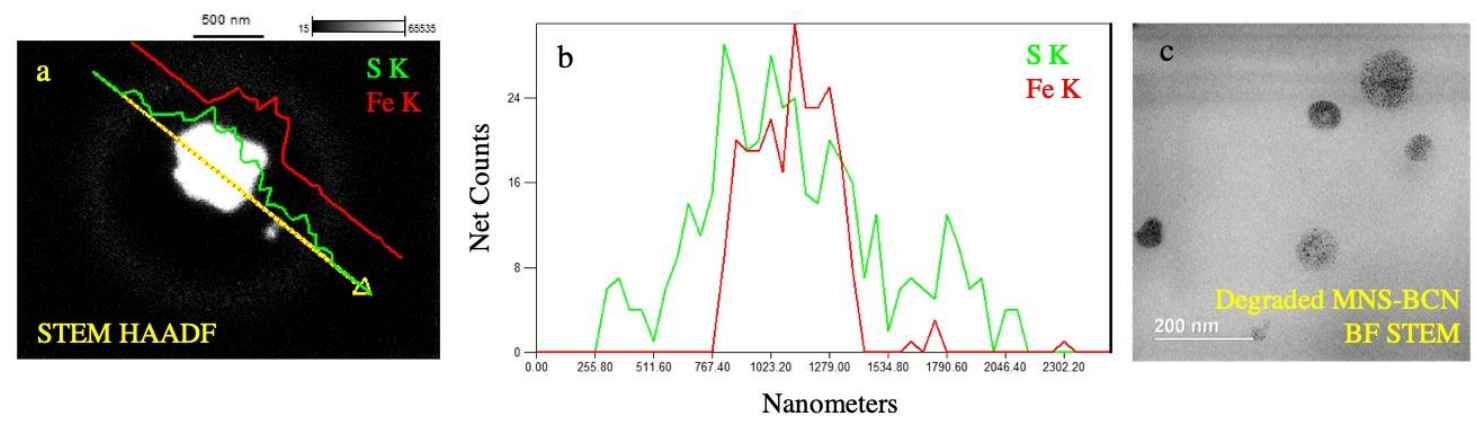

Figure 2. a) HAADF STEM images of MNS-BCN with the results of an EDS line scan projected on the image. Red refers to the quantitative iron results, while green refers to sulfur. b) A graphical representation of the line scan referenced in (a). c) Bright field STEM polymer-stabilized MNS aggregates generated by exposing BCNs to oxidative conditions.

\section{References}


[1] S Bobbala, SD Allen, and EA Scott, Nanoscale 10 (2018), p. 5078-5088.

[2] SD Allen et al, ACS Appl Mater Interfaces, 10 (2018), p. 33857-33866

[3] C Hacker et al., Scientific Reports 6 (2016), 25275

[4] S Bobbala S, SD Allen, S Yi, M Vincent, M Frey, N Karabin, EA Scott. Nanoscale. 2020. doi: 10.1039/C9NR10921H.

[5] This work was supported by Air Force Research Laboratory grant FA8650-15-2-5518, Army Research Office grant W911NF1810200 The research of Evan A Scott and Mallika Modak is supported by the National Science Foundation (CBET-1806007 and CAREER-1453576), the National Institutes of Health Director's New Innovator Award (NHLBI 1DP2HL132390-01), and the National Institute of Allergy and Infectious Disease (NIAID 1R21AI137932-01A1).This work made use of the Electron Probe Instrumentation Center and BioCryo facilities of Northwestern University's NUANCE Center, which has received support from the Soft and Hybrid Nanotechnology Experimental (SHyNE) Resource (NSF ECCS-1542205); the MRSEC program (NSF DMR-1720139) at the Materials Research Center; the International Institute for Nanotechnology (IIN); the Keck Foundation; and the State of Illinois, through the IIN. 